\title{
SNARES, tethers and SM proteins: how to overcome the final barriers to membrane fusion?
}

\author{
Herre Jelger Risselada ${ }^{1,2}$ and $\odot$ Andreas Mayer $^{3}$ \\ ${ }^{1}$ Department of Theoretical Physics, Georg-August University of Göttingen, Göttingen, Germany; ${ }^{2}$ Leiden Institute of Chemistry (LIC), University of Leiden, Leiden, \\ The Netherlands; ${ }^{3}$ Département de Biochimie, Université de Lausanne, Epalinges, Switzerland \\ Correspondence: Herre Jelger Risselada (herre.risselada@uni-goettingen.de) or Andreas Mayer (andreas.mayer@unil.ch)
}

Received: 31 July 2019

Revised: 4 December 2019

Accepted: 16 December 2019

Version of Record published: 17 January 2020
Physiological membrane vesicles are built to separate reaction spaces in a stable manner, even when they accidentally collide or are kept in apposition by spatial constraints in the cell. This requires a natural resistance to fusion and mixing of their content, which originates from substantial energetic barriers to membrane fusion [1]. To facilitate intracellular membrane fusion reactions in a controlled manner, proteinaceous fusion machineries have evolved. An important open question is whether protein fusion machineries actively pull the fusion reaction over the present free energy barriers, or whether they rather catalyze fusion by lowering those barriers. At first sight, fusion proteins such as SNARE complexes and viral fusion proteins appear to act as nano-machines, which mechanically transduce force to the membranes and thereby overcome the free energy barriers $[2,3]$. Whether fusion proteins additionally alter the free energy landscape of the fusion reaction via catalytic roles is less obvious. This is a question that we shall discuss in this review, with particular focus on the influence of the eukaryotic SNARE-dependent fusion machinery on the final step of the reaction, the formation and expansion of the fusion pore.

\section{Introduction}

The fusion of two lipid vesicles entails changes in membrane topology and lipid conformation. Membranes need to be brought into direct contact, which requires the removal of the hydration shells of the outer leaflets. It is energetically feasible to drive this apposition through the mechanical force that is exerted by fusion proteins - at least for inducing point-like contacts $[2,4,5]$. The fusion proteins are assumed to deform a small membrane area into spike-like structures that protrude towards the fusion partner and favor splaying of lipids in the outer leaflet. Spike formation and lipid splaying promote the merging of the outer leaflets, leading to a hemifusion structure. Here, lipids can pass between the outer leaflets but content mixing is still prevented by the separated inner leaflets [6-10]. Hemifusion appears to be a universal intermediate that can be observed in fusion reactions between purely lipidic bilayers as well as in protein-driven fusion between physiological membranes [2]. This hemifusion intermediate is usually considered as a 'stalk'. Other models exist, however, e.g. of fusion through proteo-lipidic hybrid structures or through entirely proteinaceous channels [5,11-15]. While these are also supported by some experimental evidence, much more data and theoretical work are available on stalk-based fusion. Therefore, we conduct our further discussion of fusion in this framework.

Full fusion requires the inner leaflets to merge. They must approach each other, which requires a stalk to be compressed and to widen $[16,17]$. The mechanical force provided by SNARE proteins is assumed to drive a local deformation (indentation) of the inner leaflets in the hemifusion structure, enforcing lipid rearrangements and generation of an initial fusion pore. This initial, small pore is in a 
local energy minimum, i.e. it is metastable and its expansion requires energy input or catalysis by lowering of the respective energy barriers [18]. Understanding how this requirement is met by fusion proteins is a major challenge and one of the central problems to be addressed by the field.

\section{Cast of characters - the SNARE-associated protein machinery}

SNARE proteins drive membrane fusion reactions inside eukaryotic cells. SNAREs from apposed membranes assemble into parallel coiled-coil complexes that force their transmembrane anchors, and thereby the two membranes, into close proximity. When assembled into complexes, SNAREs are $\alpha$-helical from their heptad repeat domains (SNARE domains) up to their transmembrane domains (TMD). They essentially constitute elastic $\alpha$-helical rods, which can transmit mechanical force onto the membranes and thereby perturb local bilayer structure.

SNARE-mediated fusion has been extensively studied, both in vivo and in artificial reconstituted membrane systems. These approaches have taught us many details about the structure and assembly of SNARE complexes and about the forces they can exert on bilayers. Numerous excellent reviews summarizing the fundamental molecular properties of SNAREs as well as of SNARE-associated SM proteins, tether proteins and Rab-GTPases have been published (see, e.g. [19-22]). We shall hence restrict our description of those to the necessary minimum and concentrate on the role that SNARE complex-associated proteins play in fusion pore opening. This question has not received sufficient attention because, for the sake of simplicity and clarity, the results from in vitro and in vivo studies are usually interpreted under the assumption that SNAREs are the sole driving force for fusion. This neglects the interactions of SNAREs with members of other conserved protein families, which are equally essential for successful membrane fusion in vivo, such as SM proteins, Rab-GTPases and tether proteins. In the following, we will discuss the energetics of fusion pore formation and expansion and focus on the question of how SNAREs and particularly their associated proteins modify the properties of the fusion site (Figure 1).

Tether proteins mediate contact between membranes. They bridge them through interactions with lipids or proteins on the two membranes [19,20]. Tether proteins usually interact with and can be activated and/or recruited to membranes through Rab-GTPases [21]. While Rab-GTPases are quite conserved at the primary sequence level, tether proteins are heterogeneous. There are highly extended coiled-coil tether proteins, characterized mainly on the Golgi, and multi-subunit tether complexes (MTCs), which exist on a variety of compartments. Despite the different composition and primary sequences of their subunits, MTCs share some common structural features. MTCs are of substantial size, many of them with a molecular mass close to the megadalton range and a size of 10-20 nm [22], as illustrated by the HOPS complex in Figure 2.

Besides Rab-GTPases, MTCs interact also with SNAREs [23]. This interaction can be direct (DSL complex; HOPS) [24], but also implicate MTC-associated SM proteins as intermediaries (e.g. in HOPS, CORVET, Exocyst). Interactions can occur with individual SNAREs as well as with partially or fully assembled SNARE complexes [25]. They may involve both the coiled-coil-forming heptad repeats and the N-terminal regulatory domains of SNAREs [24,26,27]. How these interactions evolve along the reaction coordinate of a fusion reaction remains to be resolved. It is, however, clear that Rabs, MTCs and SM proteins can strongly promote SNARE complex formation [28-32]. They may do so by a combination of several activities: by keeping the membranes at a distance that allows SNARE complex zippering; by increasing the local concentration of SNAREs at the docking zone; by actively catalyzing SNARE assembly through 'opening' the interaction of the heptad repeats with their N-terminal autoinhibitory domains (e.g. Dsl); or by keeping SNAREs 'in register', such that their heptad repeat domains can efficiently form stable SNARE complexes (e.g. SM protein Vps33). The association of MTCs with SNARE complexes can also compete with SNARE complex binding to NSF. This might protect SNAREs against NSF-mediated disassembly, shift the equilibrium towards SNARE complex accumulation [33,34], and enhance the specificity of SNARE complex formation [26,35].

The interaction of SNARE complexes with SM proteins, MTCs and Rab proteins must profoundly influence the accessibility of SNAREs, the orientation and conformation of SNARE complexes, and the topology of the membranes surrounding them. This follows from a simple consideration of the sizes of these molecules and of the steric constraints that must emanate from them (see Figure 1). SNAREs are relatively small. Even fully assembled SNARE complexes remain below $100 \mathrm{kDa}$, whereas their associated proteins are large, such as SM proteins $(100 \mathrm{kDa})$ or MTCs $(0.25-1 \mathrm{MDa})$. Thus, SNARE-associated proteins will have a major impact on the 
a

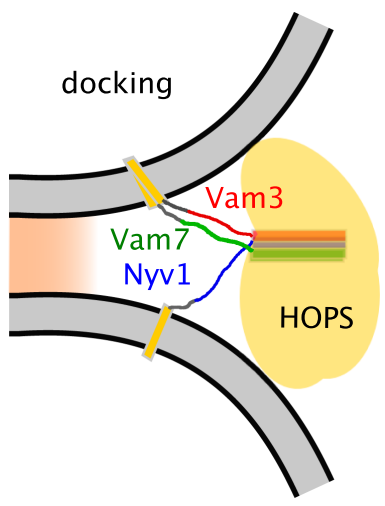

b

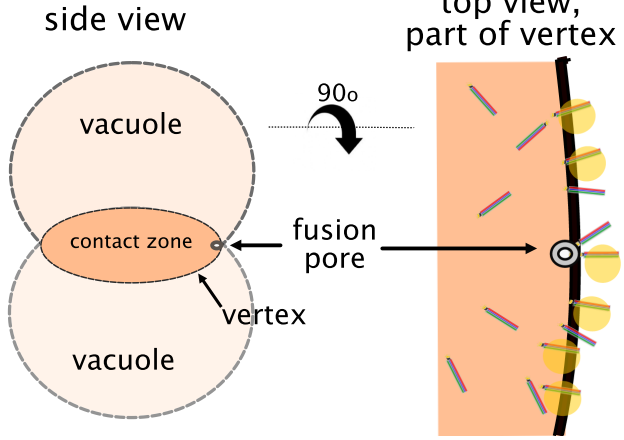

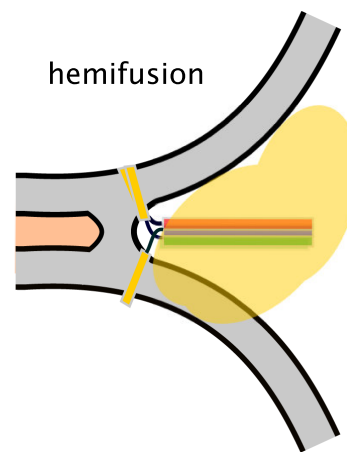

top view,

part of vertex

shape and dimensions of the holo-complex, and on the conformational changes and forces that it can impose on the two membranes to be fused (Figures 1 and 2).

\section{SNARE-mediated opening of the fusion pore}

The SNARE complex forms by the stepwise association of the heptad repeats from their $\mathrm{N}$ - to their C-terminal ends ('zippering'). Thereby, these 'SNARE domains' transit from a partially unstructured conformation into a fully folded coil-coil [36-39] (Figure 1a). Due to the rigidity of the $\alpha$-helical linker region between the heptad repeats and the TMDs of the SNAREs, zippering pulls the TMDs into closer proximity and finally aligns them with each other. When the two parts of the SNARE complex reside in separate membranes, or in a hemifusion structure, the TMD hence exerts force on this membrane. Forces generated by the fully zippered neuronal SNARE complex at the C-termini of the SNARE domains have been experimentally estimated by applying 
a

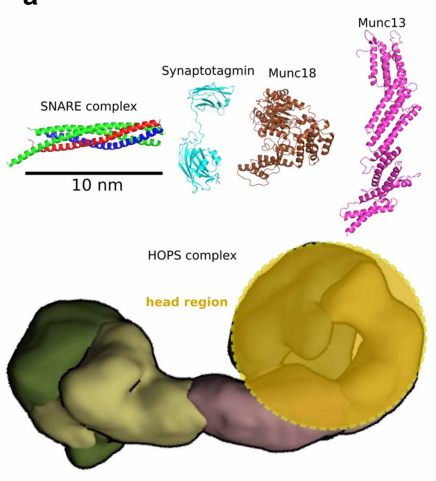

b 3 SNARE complexes

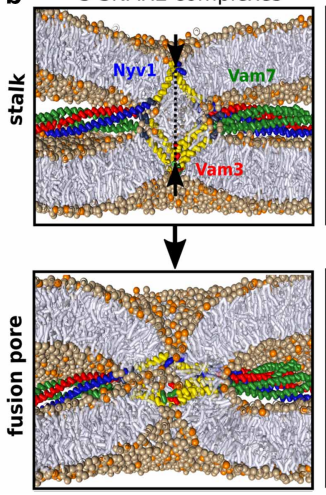

+HOPS (head region)

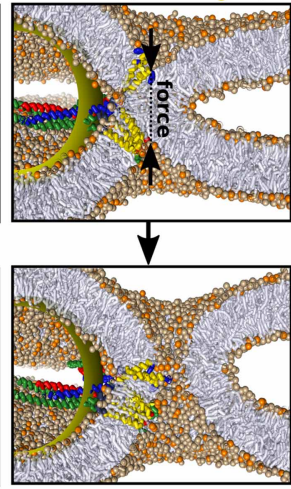

C

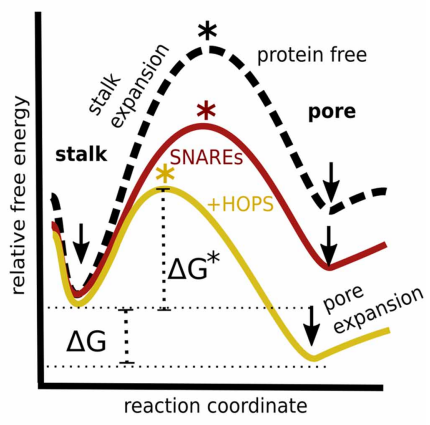

Figure 2. Increasing the volume of SNARE complexes through associated proteins.

(a) Size comparison of the cytosolic part of a SNARE complex with the different SNARE-associated proteins. All proteins and the tethering complex HOPS are represented on the same scale. The size and shape of the HOPS complex is based on cryo-EM densities (adapted from [74]). The SNARE-binding region of HOPS, i.e. the head region which encloses the Vps33 subunit, is encircled in yellow. Although cryo-EM studies performed under different conditions yielded different shapes for the complex [74,108], an approximation of its SNARE-binding 'head' region as a $14 \mathrm{~nm}$-sized sphere is justified, based on the known crystal structure of the Vps33-Vps16 complex, which constitutes the major part of it [109]. (b) Opening of the fusion pore in the presence of three vacuolar SNARE complexes from yeast. The SNARE complexes impose force on the stalk via the C-termini of the TMDs (black arrows). This force leads to thinning (approaching the lumenal leaflets) of the stalk and to its evolution into a fusion pore. The geometry imposed on the fusion site by the head region of HOPS, which is located nearby, eases non-leaky indentation and lateral widening of the stalk. Shown is a cross-section through the fusion site, perpendicular to the plane of the membranes. Fatty acyl chains of the lipids are shown in gray, the lipid headgroups in orange and beige, and the SNARE-binding region of HOPS is approximated through a yellow ball of corresponding dimensions (as indicated in a). All SNARE TMDs are colored in yellow, their hydrophilic regions in red, blue and green, as indicated. The images have been extracted from a simulation run. The same color scheme is used in all the following figures. Due to the movement of SNARE complexes, not all complexes are visible on all cross-sections. (c) Kinetics of the stalk to fusion pore transition. Each curve represents the reaction pathway of minimal free energy - the most likely reaction pathway - on a high-dimensional (hyper) surface, i.e. the so-called free energy landscape (free energy is a function of the co-ordinates and momentum of all particles). This representation is reduced to two dimensions by projecting this high-dimensional landscape on a one-dimensional reaction co-ordinate (e.g. the thickness of the stalk). The rate $k$, at which this (forward) transition occurs, is $k=\mathrm{Ae}^{-\Delta G * / k_{\mathrm{B}} T}$, with $\mathrm{A}$ being the kinetic pre-factor, $\Delta G^{*}$ being the free energy barrier, $k_{\mathrm{B}}$ the Boltzmann constant and $T$ the temperature. Although fusion proteins may alter the pre-factor $A$, the rate of the transition is dominated by the much larger exponential factor comprised by the free energy barrier $\Delta G^{*}$. SNARE complexes can lower $\Delta G^{*}$ and increase the stability of the fusion pore with respect to the stalk $(\Delta G)$ by actively imposing force along the reaction co-ordinate. HOPS catalyzes the SNARE-mediated stalk-pore transition by (indirectly) perturbing the structure/nature of the reactant (stalk), the barrier and the product state (fusion pore).

optical tweezers, yielding a value of $17 \mathrm{pN}$ to reversibly fold/unfold the SNARE domain [37]. It is, however, unclear how this force is transduced to the C-termini of the TMDs, i.e. the part that can actively work on the trans-leaflets of the bilayer to drive the evolution of a hemifusion intermediate, open and expand a fusion pore. That the TMDs must transmit force at this point is supported by the observation that SNAREs with a truncated TMD or with a lipidic membrane anchor, which span only half of the bilayer, are inefficient in opening fusion pores [6,40-44]. Interestingly, the fusion activity of SNARE complexes carrying a lipid anchor can be stimulated in vitro by the addition of an excess of SNARE-associating proteins, such as the MTC HOPS [45].

The force that SNARE complexes can exert on fusion intermediates depends on the adopted secondary structure of the linker regions connecting the SNARE domain to the TMD. Coarse-grained simulations suggest that the SNARE complex of yeast vacuoles transduces a force of $18 \mathrm{pN}$ to the trans-leaflets of an $\sim 8 \mathrm{~nm}$ thick fusion stalk (see Figure 3a), when all three of its TMD-containing SNAREs (Nyv1, Vam3 and Vti1) are $\alpha$-helical [46]. However, the magnitude of this force is halved to $9 \mathrm{pN}$ when the linker of Vtil adopts a nonhelical structure [46]. The vacuole SNARE complex with its three TMDs might hence generate larger pull forces than the neuronal SNARE complex, which is anchored by only two TMDs. This different topological 

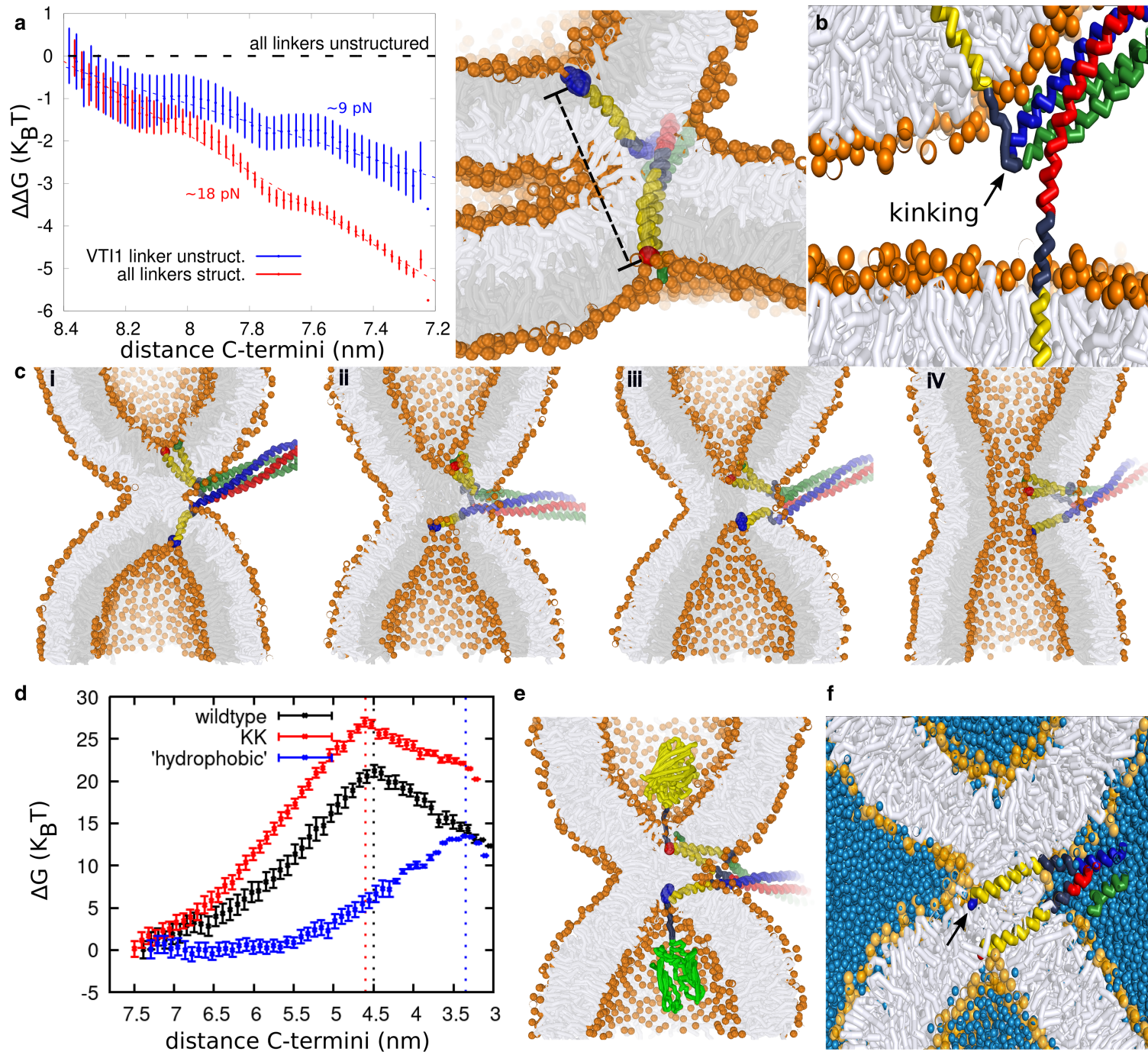

$\mathbf{f}$

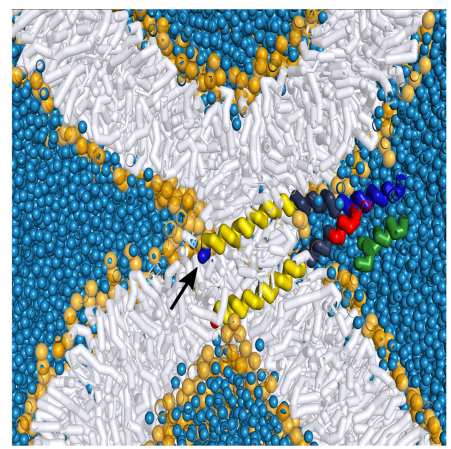

Figure 3. SNARE-mediated forces drive the stalk to fusion pore transition.

(a) Estimation of the force exerted on the stalk by C-termini of the vacuolar SNARE TMDs as they approach each other, based on coarse-grained molecular simulations $[46,75]$. The distance displayed in the graph is illustrated by a dashed line in the cross-section through the fusion site that is shown next to the graph. Values are shown for three different SNARE complexes: With the linker regions between the TMDs and SNARE domains of all three membrane-anchored SNAREs $\alpha$-helical (structured) or non-helical (unstructured), or with only a single linker region (of the Vti1 SNARE) non-helical. (b) Zipping with a SNARE that has prematurely attained its $\alpha$-helical, structured conformation results in excessive kinking of the complementary SNARE, which is still partially unstructured. Shown is a close-up of a partially zippered SNARE complex linking two adhering membranes. (c) Indentation of the stalk through the SNARE C-termini results in the formation of a fusion pore. Shown is a cross-section through a stalk (I), in which the inner leaflets progressively approach each other, driven through a force applied on the C-termini of the SNAREs (II-III), until a fusion pore opens (IV). (d) Free energy profile of the stalk-pore transition for different chemically modified C-termini, based on coarse-grained molecular simulations [46,75]. Simulations have been performed as illustrated in c, using SNAREs in which the C-terminus carried either short charged (Lys-Lys) or hydrophobic extensions, or no extension. Vertical dotted lines indicate the location of the barrier to pore opening for the three cases. (e) Two extremes: in an experiment as in (c), large fluorescent protein tags attached to the SNARE C-termini do not interfere with the stalk-pore transition, as long as only a single SNARE complex is opening the pore. When several SNARE complexes collaborate to indent the stalk, such tags strongly interfere because they sterically interfere with a concentration of several C-termini in the indented region [46]. (f) 'Misfiring' of the SNARE complex can occur when the SNARE C-termini are too hydrophobic and/or when the free energy barrier to fusion pore formation is too high. Shown is the cross-section through a fusion stalk in which the C-terminus of one SNARE has been pulled through the bilayer, ending up in the buffer surrounding the stalk. 
feature may be an adaptation to a larger free energy barrier of fusion pore opening in vacuole fusion. The barrier is expected to be higher due to the lower membrane curvature of vacuoles in comparison with synaptic vesicles, which renders membrane curvature at a fusion site less compatible with the formation of a fusion pore (see below).

During SNARE zippering, the SNAREs need to transit from an unfolded conformation into a continuous $\alpha$-helix and to bend at the same time [47,48]. If this transition is not well co-ordinated for both sides of a SNARE complex, the folding process might be hung up in a non-productive state [48]. Premature folding of only one of the SNAREs into an $\alpha$-helical rod may force the linker region of the other half of the SNARE complex into an overly bended conformation, which may hinder it from ever adopting a continuous $\alpha$-helical structure (see Figure 3b) [17]. Within such a scenario, the stiffer helical SNARE (provided that its SNARE domain is not clamped to the membrane by lipid anchors or interactions with other proteins) can release its bending stress by kinking the more flexible, partially unstructured SNARE. It thereby (i) arrests the progression of SNARE zippering and (ii) impairs efficient force transduction form the SNARE domains to the TMDs. This may explain why mutations in the TMD region of synaptobrevin-2, which actually enhance helicity and stiffness of the linker in molecular simulations [49], nevertheless reduce the experimentally observed fusion activity [50]. Efficient force transduction from SNARE complexes to fusion intermediates may, therefore, require a timed and regulated adoption of $\alpha$-helical structure - here lies an interesting potential role for SNARE-associated proteins (SM and tether proteins), which may guarantee exactly this when they catalyze SNARE complex formation [30,32,51-53]. That SNARE-associated proteins such as the SM protein Munc18 shift the equilibrium from a half-zippered intermediate towards the fully zippered complex, as evident from FRET measurements in reconstituted nanodisc systems $[48,54]$, can also be understood in this context.

The X-ray structure of the complete neuronal SNARE complex, which might represent the post-fusion state, suggests that not only the SNARE domains but also the TMDs might fully associate [55]. This might provide an additional release of free energy during fusion. However, in order to access this potential driving force already during hemifusion, the TMDs would have to provide sufficient mechanical flexibility to allow a gradual zippering of the TMDs. This notion may explain the existence of conserved TMD residues in the synaptic SNARE synaptobrevin, which easily break helicity and are relevant for fusion pore opening [56]. An active role of the TMD is consistent with the observation that single amino acid substitutions in the TMDs of syntaxin and synaptobrevin influence fusion pore conductance and dynamics [13,56-58] and that the native TMD of synaptobrevin is required to allow efficient content release in a synthetic fusion system [59]. However, it remains unknown whether these substituted SNAREs are still sorted efficiently to the site of exocytosis in vivo. This poses a caveat because manipulations of the TMD can result in miss-sorting [43]. On the other hand, the TMDs of all vacuolar SNARE proteins could be exchanged for TMDs from proteins unrelated to fusion without a significant impact on the fusion of yeast vacuoles [43]. This argues against evolutionarily conserved, sequence-specific roles of SNARE TMDs that go beyond those of a mechanical membrane anchor. It remains an important and open question to which extent conserved residues or sequence features in the SNARE TMDs have been genetically imprinted in response to the energetics of the different fusion barriers, or by other aspects relevant to SNARE function, such as their biosynthetic sorting, their re-activation after fusion, or their recycling to their compartment of origin.

Recent coarse-grained simulations suggested that the TMDs of neuronal SNAREs are not inert with respect to the barrier to hemifusion, i.e. the formation of the stalk. They can induce a substantial reduction $\left(\sim 10 k_{\mathrm{B}} T\right)$ in the free energy of both the stalk and of the barrier against its transition into a pore [60]. This effect may be relatively sequence-independent but relate to the effective hydrophobic length of the TMD. The SNARE TMD can display a hydrophobic mismatch with the membrane, such that its length is better accommodated in a stalk than in a simple bilayer [60].

The C-termini of the TMDs may be critical to allow the SNARE complex to drive the evolution of a hemifusion intermediate such as a stalk. The hydrophilic nature and the net charge of the C-termini enable indentation of the stalk because they oppose the transition of the C-termini into the hydrophobic membrane core, which would otherwise perforate the bilayer [46,61] (see Figure 3c,d). TMD-induced indentation compresses the stalk, i.e. it reduces the distance between the two lumenal leaflets (stalk thickness), and it simultaneously widens it parallel to the membrane surfaces. An example of such membrane remodeling by the C-termini is demonstrated in Figure 3c. The addition of further SNARE complexes to the site of membrane fusion must be expected to incrementally reduce the thickness of the stalk, bringing the stalk closer to its barrier against fusion pore opening until a sufficient thermal fluctuation enables sudden barrier crossing. The attachment of large 
hydrophilic peptide tags to the C-termini of vacuolar SNAREs (Figure 3e), which renders their membrane penetration energetically very costly and highly unlikely, does not impair fusion of yeast vacuoles [46]. This strongly suggests that fusion does not rely on 'perforation' by the C-termini but rather involves indentation and associated remodeling of the membranes.

Nevertheless, chemical alterations and substitutions at the TMD C-termini can alter the kinetics of the fusion of exocytic vesicles (of $\sim 100 \mathrm{~nm}$ diameter) [61]. Such modifications retard fusion when the hydrophobicity of the C-termini is decreased, or accelerate fusion when hydrophobicity is increased [62]. These effects can be reconciled with an indentation mechanism for fusion pore opening if we take into consideration that the local chemical environment at the C-termini contributes to the energetic cost function of indenting a fusion intermediate. For example, adding an additional KK motif to the C-termini of neuronal SNAREs (or mimics thereof) [61] increases the indentation force and total energetic cost of indenting the stalk (Figure 3d). However, barrier crossing itself occurs at a slightly smaller stalk indentation (vertical dashed lines in Figure 3d). The increase in work is associated with the steeper slope of the curve (the responsive force against indentation). This suggests that the KK motif builds up more stress in the stalk structure than the wild-type when the C-termini come together. However, the free energy of the compressed stalk becomes competitive to the free energy of the stalk barrier 'faster', i.e. at a shallower indentation. In contrast, increasing the hydrophobicity of the C-termini displaces the barrier towards a more profoundly indented state but simultaneously reduces the indentation force (the slope of Figure 3d) and the total energetic cost of indentation. Since the force transmitted by the TMDs probably remains unaltered, fusion rates can then be improved by shaving off the energetic cost (force) of stalk indentation via chemical alterations of the C-termini. When the C-termini become too hydrophobic, however, a perforation threshold can be reached, at which the SNARE complex 'misfires', i.e. it zippers and pulls its TMD through the membrane without driving the fusion reaction (see Figure $3 \mathrm{f}$ ). It is then possible that the SNAREs offering the fastest fusion kinetics are the ones that reduce the cost of indentation to such an extent that it allows a substantial amount of 'misfiring' with only an occasional 'hit'. Such properties may be non-optimal for some in vivo fusion reactions when, as for example in regulated exocytosis in neurons, high reliability and temporal fidelity of fusion are indispensable.

\section{Pumping up the volume!}

The free energy of a hemifusion structure strongly depends on its shape and to which degree this shape is compatible with the arrangement and chemical properties of its lipids and proteins [63]. Steric effects at the fusion site hence deserve attention. Since, as outlined above, SNARE complexes associate with a variety of other proteins, which in their sum are often much larger than the SNARE complex itself (Figure 2), it is not sufficient to consider only the influence of isolated SNARE complexes on the fusion site. Proteins associated with the SNARE complex can modify the distance and curvature of the membranes at the fusion site and can thus have a significant impact on the energetic landscape of fusion pore formation and expansion. In line with this, the vacuolar tether protein complex HOPS, its associated SM protein Vps33 and the exocytic SM protein Munc18-2 are necessary for the transition from hemifusion to full fusion [64-66]. Also, the SM protein Munc18-1 influences fusion pore dynamics in exocytosis [64-66] and stimulates SNARE-dependent liposome fusion [31]. SNARE-associated proteins, such as Munc18 and the exocytic calcium sensor synaptotagmin, were also proposed to scaffold several SNARE complexes around a fusion site and favor their synergistic action on it [67-70].

Tether complexes interact with membranes through protein-protein interactions, e.g. with Rab-GTPases, and through a direct affinity for membrane lipids, as exemplified by the vacuolar tether/SM protein complex HOPS, which binds acidic phospholipids [19,71-74]. While these interactions can keep membranes in proximity to each other, there is no evidence that tether proteins actively deform membranes at a fusion site. In contrast, SNARE complexes are able to actively generate the essential curvature near the fusion site and force the membrane into close apposition. In doing so, they will 'parachute' the membrane on top of the much more voluminous tether and SM proteins, which are associated with the SNAREs (Figures 1 and 2). While this increases the work required to bring the membranes in close apposition and form a fusion stalk, this work can be reduced to some extent by favorable interactions of these voluminous proteins with the membrane. In the case of the tether/SM protein complex HOPS, for example, such favorable interactions would be provided by two Rab-GTPases and acidic lipids, which cooperatively enhance the affinity of HOPS [19,71-74] for the membrane. Whereas such favorable membrane interactions may reduce the energetic cost, they are not essential for stimulating fusion. This follows from molecular dynamics simulations and from the experimental observation 
that fusion pore formation is strongly accelerated by associating the SNARE complex with soluble proteins, which have no affinity for the bilayers but increase SNARE complex size and deform the fusion site [75].

Trans-SNARE complexes exert force on the membranes and deform them. To reduce the energetic cost of this membrane deformation, SNARE complexes can accumulate at the curved edge of the membrane-membrane contact zone (Figures $1 \mathrm{~b}$ and 4). In support of such a notion, SNAREs and the tether/SM protein complex HOPS of vacuoles were found to concentrate at the edge of the contact zone in a vertex ring [76-78]. Similar vertex enrichment of (non-SNARE) mitochondrial fusion proteins has also been observed by cryo-electron tomography of contact zones between mitochondria fusing in vitro [79]. Recent in vivo observations by fluorescence microscopy suggest that the fusion site between two yeast vacuoles is located near the edge of this contact zone (see Figure 4) [80]. Association of the SNARE complex with voluminous protein complexes will drive SNARE complexes even more effectively towards the edge of the contact zone, where this volume can most easily be accommodated.

The formed SNARE/HOPS complexes can be understood as a space-filling, molecular gas in two dimensions (the membrane surface). Restriction of SNARE/HOPS complexes at the docking zone or vertex imposes an entropic pressure (crowding force) that drives the expansion of the contact zone [81]. This entropic pressure is expected to scale with the (local) concentration of SNARE complexes (like an ideal gas) and with the volume (size) of each complex (Lager 'crowders' reduce the available configurational space) [82]. If HOPS, because of its steric volume, confines its associated SNARE complexes to the vertex region, it effectively increases the local concentration of SNARE complexes and the concomitant entropic pressure [81], which can additionally enforce an expansion of the contact zone. Therefore, voluminous SNARE complexes likely induce larger contact zones than 'skinny' SNARE complexes, illustrating how SNAREs can be put in a 'higher gear' when their volume is being increased via association with tether and SM proteins (Figure 1).

\section{Effects of SNARE-associated proteins on the energetics of the stalk-pore transition}

The presence of bulky SNARE complex ligands dramatically enhances the capacity of SNARE complexes to open a fusion pore. This could be demonstrated through the in vitro fusion of yeast vacuoles. In the absence of the tether/SM protein complex HOPS vacuoles can form trans-SNARE complexes to similar levels as wild-type

a

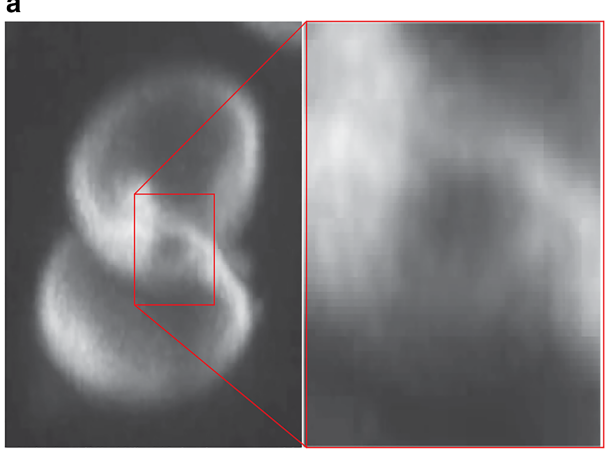

b

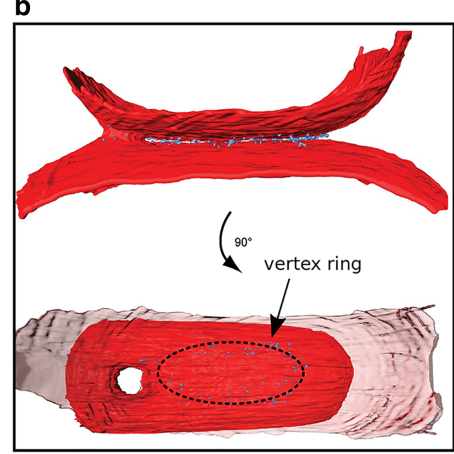

c

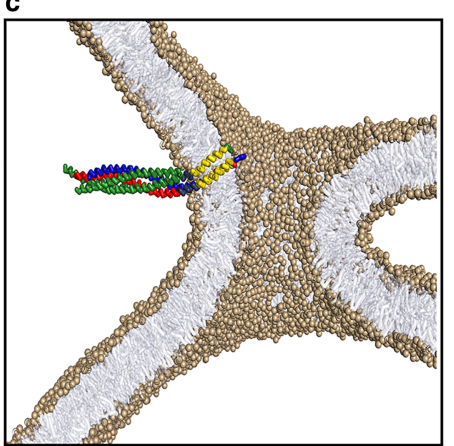

Figure 4. Fusion occurring within an extended contact zone.

(a) 3D-reconstruction of confocal fluorescent image stacks of a fusion pore formed between to vacuoles in a living yeast cell. Notice its location near the edge of the contact zone. Only two large vacuolar compartments, which are located within a single yeast cell and adhering to each other, are visible due to their staining with a fluorescent vital dye. The rest of the cell and the cell wall are not visible. Adapted from [80]. (b) Electron cryo-tomography imaging of a fusion pore and contact zone between two mitochondria fusing in vitro. The dashed black ring indicates the vertex ring around the contact zone, which is enriched in fusion proteins, in this example mitofusins (marked by blue dots). The images show cross-sections through the contact zone perpendicular to it (upper image) or parallel to the contact zone (lower image). Adapted from [79]. (c) Molecular simulation of an edge fusion pore, performed as described [75]. The edge pore is radially asymmetric, and its overall shape and structure depend on the contact angle between the vesicles. Shown is a cross-section of a fusion pore at the vertex of a contact zone, cut perpendicular to the contact zone. 
vacuoles, but these SNARE complexes can bring the membranes only into hemifusion [75]. The addition of HOPS then drives the reaction to the pore opening. Strikingly, fusion pore opening can be stimulated to the same degree when HOPS is replaced by other, artificial SNARE-binding proteins, such as antibodies to the SNAREs. The only specific property required for stimulation of fusion is that these artificial ligands add a similar volume to the SNARE complex and bind it in close proximity to the site of membrane fusion, on or close to the SNARE domain. This provides strong experimental evidence that steric constraints imposed on the fusion site by SNARE-associated proteins - in the physiological setting mostly tether and SM proteins provide a major driving force for fusion pore formation.

Coarse-grained molecular simulations and continuum models could be used to explore the origins of this strong stimulation of fusion [75]. These approaches revealed that the increased volume provided by non-SNARE proteins at the fusion site greatly reduces the energy barrier that the stalk must cross in order to evolve into a fusion pore. This is due to the increased curvature that these proteins impose on the stalk (Figure 2b), which partially 'anticipates' the curvature of a later fusion pore [83]. It thus reduces the work required to attain it. The work is provided by the SNAREs, which convert the strain produced by trans-SNARE pairing into a force acting on the C-termini of their TMDs, as outlined above. This force progressively indents the lumenal leaflets until a fusion pore develops. Reaching the critical indentation, from which a fusion pore opens spontaneously, requires less compression of the stalk and therefore less work in the presence of bulky SNARE-associated proteins.

Bulky SNARE ligands will not only impose curvature on the fusion site directly. They are expected to have further effects by which they accelerate fusion. They will drive the SNAREs, and the fusion site that they are located in, towards the edge of the contact zone. The membrane bending that occurs here imposes additional curvature on the stalk to further promote fusion pore formation (Figure 4c). Bulky SNARE ligands also exert a peristaltic force on the SNARE complex, which drives the complex away from the fusion site and generates the additional pulling force on the C-termini in the indented stalk. Furthermore, it was proposed that bulky SNARE ligands could dictate a twisted positioning of the SNARE domains at the fusion site [84]. Thereby, they might allow the SNARE complex to zipper by up to half a turn further than it normally would in a 'relaxed' state, i.e. when the rotational positioning of the SNARE domains is not restrained by associated proteins. Also this would provide an additional pulling force on the TMDs.

\section{Nanoscopic fusion pores can be long-lived}

Fusion pores are metastable. They can flicker, expand into full fusion or revert into a hemifused state [5,85]. The pores are usually thought of as symmetric hourglass-shaped lipidic structures lined by fusion proteins. Expansion of the fusion pore is opposed (I) by a free energy cost associated with extending its curved membrane perimeter, and (II) by creation of excess membrane area during pore expansion (growth of vesicle volume). SNARE-mediated fusion pores have been particularly well characterized in exocytosis, where they are easily accessible to high-resolution electrophysiological measurements, and in synthetic systems using nanodiscs. While SNARE-dependent fusion pores are generally considered as transient structures, with lifetimes in the sub-second range [86,87], recent in vivo analyses in yeast showed that the vacuoles in this organism are connected by nanoscopic fusion pores and that this state is quite stable (for many minutes) [80]. These pores do not allow passage of small $(0.25 \mathrm{kDa})$ soluble fluorophores, indicating that they are surprisingly narrow with a diameter of $1 \mathrm{~nm}$ or less - and may not expand for a long time. Their existence could only be shown through fluorescent lipid markers integrated selectively in the inner membrane leaflet, and through the fact that they allow passage of TMDs spanning both leaflets (Figure 5a). Whether comparably stable nanopores exist in other SNARE-mediated physiological fusion steps, where they might be considered as hemifused states [88], is currently unknown because such pores may not be easily revealed by the passage of soluble content markers. Ironically, one may hence coin these pores 'black holes' - because they easily escape detection in optical assays and - as explained below - their formation may be related to the 'collapse' of a fusion pore.

An analysis of the properties of such nanoscopic fusion pores by coarse-grained molecular dynamics simulations provided some insight into factors that influence their stability and expansion [80]. The insertion of three vacuolar SNARE complexes into a stalk at the fusion site yielded a pore of $3 \mathrm{~nm}$ diameter (Figure 5b). Further expansion of the pore is opposed by a free energy cost associated with extending the interfacial length of the highly curved circumference of the pore [83]. It would, therefore, require the presence of an external force, such as the presence of osmotic pressure or a growing protein coat on the neck of the fusion pore [89-94]. As an alternative, recent studies on the SNARE-mediated fusion of nanodiscs indicated that incorporation of 

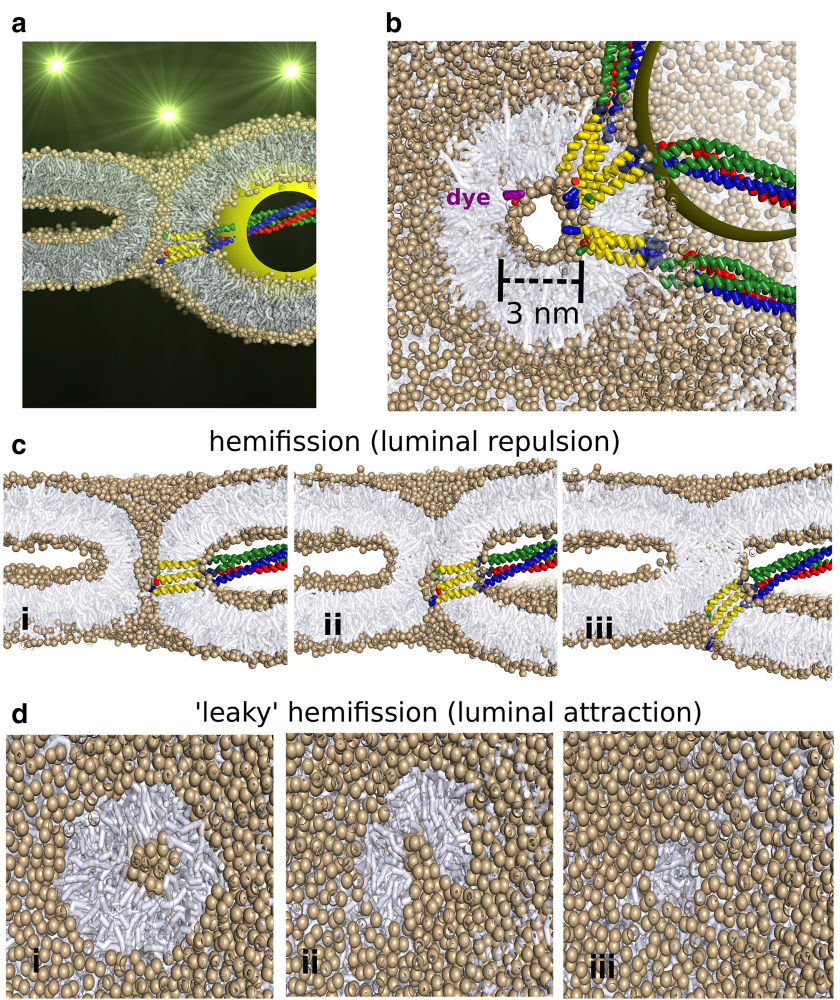

Figure 5. Nanopores and their stability.

(a) Artistic representation of a 'black hole': mixing of soluble dye molecules between the separate compartments does not occur despite the presence of a fusion pore. Shown is a non-expanding fusion pore in cross-section, with a single SNARE complex integrated and a ball representing the SNARE-binding part of HOPS bound to it. Soluble dye molecules are shown in light green. (b) A tension-less fusion pore observed in molecular dynamics simulations in the presence of three vacuolar SNARE complexes and the SNARE-binding region of HOPS, bound as a ball to one of the SNARE complexes. Shown is a cross-section through the pore, parallel to the membranes in the contact zone. The purple molecule indicates a fluorescein molecule (MW 332Da) and the dashed line the diameter of the pore. Adapted from [80]. (c) External compression of the pore in the presence of lumenal (hydration) repulsions results in hemifission. Shown is a pore cross-section perpendicular to the contact zone. The compression of the membranes stimulates spontaneous pore closure, which drives the SNARE complex out of the pore region and tilts it, generating strain. Adapted from [80]. (d) An adhesive force within the pore impairs hemifission. Cross-section of a fusion pore parallel to the contact zone (left). External compression of the pore eventually results in rupture (leakage) of the neck of the pore (middle) and the pore finally 'regresses' into a stalk (right).

increasing numbers of SNARE complexes into the membrane surrounding the pore incrementally increases the conductance of the fusion pore. In this case, steric repulsions between the growing number of SNARE complexes may enforce the widening of the pore [95]. Since, in vivo, nanoscopic fusion pores between yeast vacuoles persist and remain too small to passage soluble dye molecules, the physiological pool of trans-SNARE complexes and other docking factors surrounding these pores appears to remain insufficient to substantially widen the pore through this mechanism. Furthermore, it is important to highlight that there are structural differences between the fusion pores in the two experimental systems. Vacuolar fusion pores likely show axial asymmetry, because their SNARE complexes drive them to the vertex of the microscopically sized contact zone (see Figure $4 \mathrm{a}, \mathrm{c}$ ). In contrast, a pore in a nanodisc is surrounded by polymer-coated free membrane edges and, due to its tiny size ( $<25 \mathrm{~nm}$ diameter), cannot even permit the formation of an extended contact zone [85].

Interestingly, a tension-less fusion pore observed in simulations showed a diameter of $3 \mathrm{~nm}$ [80]). Such a diameter would easily permit passage of a small soluble fluorophore (Figure 4), to which the experimentally observed 'black holes' have been impermeable. These fusion pores between vacuoles must hence be of substantially smaller size $(<1 \mathrm{~nm}$ diameter) and/or restrict diffusion through the pore via other means, for example by reducing water dynamics through molecular crowding in the tiny pore [96]. What can stabilize such tiny fusion 
pores against reclosure? Pore closure increases bending stress on the bilayers [83]. It also coincides with the unfavorable dehydration of lipid head groups that is necessary to overcome hydration repulsion between the lumenal leaflets [97]. At pore sizes $<1 \mathrm{~nm}$, hydration repulsions would dominate the free energy of the pore. This suggests that the in vivo fusion pore must be subjected to an additional 'compressive' force that compensates lumenal hydration repulsion. The precise nature of this force is unclear at present. A possible explanation is the presence of effective attractions (likely of electrostatic nature) within the lumen of the pore. They might occur between charged lipid head groups, charged residues in the lumenal C-termini of the SNAREs, and divalent cations [5]. Lumenal attractions should facilitate the 'collapse' of a pore. However, they should impair the formation of a hemifission intermediate because hemifission would decrease the number of now favorable head group interactions within the interior of the pore. The free energy barrier against hemifission can be further raised when voluminous proteins decrease the curvature of the rim of the fusion pore (Figure 5a). In the presence of attractions in the pore lumen, 'hemifission' - when it is enforced via an externally applied contractive force - only occurs via an alternative asymmetric, leaky pathway [98] that circumvents 'collapse' of the fusion pore (Figure 5d). In this pathway, the free energy barrier against hemifission is in fact determined by the rupture limit of the membrane. In contrast, applying an external contractive force in the presence of luminal repulsions (e.g. through hyper-osmotic shock or inter-leaflet tension) stimulates non-leaky hemifission (Figure 5c).

\section{SNARE complex positioning}

It is paradoxical how a compressed pore can remain stable for many minutes without escaping into an energetically favorable different topology, i.e. forming a hemifission intermediate. The presence of SNARE complexes itself may 'safeguard' the pore against closure. Whereas fusion pores induced by a single SNARE complex in lipid nanodiscs readily re-close, their stability increases when additional SNARE complexes are integrated and their diameter widens $[59,86]$. The stabilization and widening of the pore could result from a radial (entropic) force that multiple SNARE complexes could exert when grouped around a fusion pore [59]. However, steric effects can also become important here. When a fusion pore re-closes, the C-termini must be pushed out of the pore, which requires to tilt the SNARE complex [80]. This tilting is opposed by the stiffness of the helical SNARE bundle, which then collides with the apposed membranes in the contact zone. If, in addition, we take into account the association of SNARE complexes with tether/SM proteins, which themselves are fixed between the membranes in the contact zone, it is conceivable that a movement of the SNARE C-termini out of the pore might be obstructed. This 'immobilization' of multiple hydrophilic SNARE C-termini within a fusion pore should provide a strong obstacle to its reclosure. That restriction of SNARE complex mobility might be important in opening and stabilizing the fusion pore is consistent with molecular dynamics simulations showing that positionally restrained SNARE complexes are effective in fusion pore opening whereas they produce hemifusion diaphragms when left free to move [99].

\section{Fusion pores of small and large vesicles face different challenges}

It is not self-evident that the parameters relevant to efficient fusion are entirely overlapping between exceptionally small exocytic vesicles, which are optimized for rapid and temporally well-controlled fusion, and larger vesicles or organelles, where the energetic barriers and concomitant indentation forces are expected to be much larger. A fusion site in the contact zone between two very large vesicles is located between two approximately flat membranes, which leaves little space for wider movements of SNAREs and their associated proteins perpendicular to the membranes. Due to their very high membrane curvature, fusion sites between very small vesicles (e.g. synaptic vesicles, diameter $<40 \mathrm{~nm}$ ) provide much more space for SNARE complexes to reorient themselves. This may be an important factor reducing fusion pore stability because it may allow SNARE complexes to diffuse out of the fusion pore more easily.

Another relevant difference concerns the size of the contact zone. Whereas this zone can be very large between docked organelles (e.g. several $\mu \mathrm{m}^{2}$ for yeast vacuoles), synaptic vesicles make rather point-like contacts $[100,101]$. For such small vesicles, the size of the early fusion pore already occupies a significant fraction of a potentially present adhesion zone, whereas this beneficial relative offset vanishes when the adhesion zone adopts a microscopic length scale. Furthermore, the minimization of curvature stress through fusion of small vesicles can drive the expansion of the fusion pore because their curvature stress can be large and competitive with the line tension of the fusion pore. Thereby, very small vesicles should be predisposed for a rapid progression 
towards fusion pore opening and expansion. 'Safeguarding' the pore against reclosure through the immobilization of SNARE complexes would thus be expected to be less important for synaptic vesicles. Yeast vacuoles, by contrast, are large and natively controlled by osmotic pressure. Safeguarding pores between clustered vacuoles is of functional benefit since a pre-existing fusion pore allows rapid adaption to changing osmotic pressures and environmental conditions [80].

Osmotic pressure and membrane tension are obvious factors that can expand fusion pores [89-94]. For vesicles adhering over a larger contact area, these can be generated by the formation of an extended contact zone via protein-mediated adhesion, which alters the volume-to-surface area ratio of the vesicles (see Figure 4a,b). Such adhesion-mediated pressure may to some degree be reduced through water exit, since membranes are quite permeable to water. However, the decrease in vesicle volume through docking also increases the concentration of physiological osmolytes inside the vesicle, thereby limiting water efflux. Docking thus induces a persistent osmotic pressure and concomitant membrane tension which can promote fusion pore expansion. The 'gearing' of SNARE complexes through bulky ligands might enhance this process. Being driven to the vertex of the docking zone more forcefully, voluminous, 'geared' SNARE complexes may thus enforce the formation of larger contact zones, build up more membrane tension, accelerate the growth of the contact zone and allow the generated tension to promote fusion before it can relax.

In contrast with very small vesicles, which make only point-contacts at the fusion site, the extensive contact zone between large vesicles should lead to an inhomogeneous probability of forming a fusion pore, already because components of the fusion machinery accumulate near the vertex. In addition, molecular simulations suggest that pores favorably break symmetry when formed at the vertex of an extended contact zone, resulting in a radially asymmetric 'edge fusion pore' (Figure 4c). This symmetry break is driven by a mutual reduction in membrane bending energy for both the fusion pore and the curved membrane edge associated with the fusion pore. Therefore, the presence of an extended docking/adhesion zone has interesting consequences for the preferred location, structure and further expansion of a fusion pore. Quite in contrast with a radially symmetric fusion pore, the expansion of an edge fusion pore is additionally governed by an effective adhesive interaction between the pore and the highly curved membrane at the edge of the contact zone. The physical principle of such an expansion is analogous to the expansion of a rim-pore formed within a hemifusion diaphragm [102]. The larger the bending free energy of the vertex, the more favorably the vertex will be replaced by part of the fusion pore. Its curvature, however, is directly determined by the apparent contact angle between the adhering vesicles, i.e. by the relative size of the docking zone. This principle might provide an explanation of why attachment of voluminous proteins to SNARE complexes can push a fusion reaction from arrested hemifusion all the way up to complete expansion of the fusion pore, as observed through the fusion of yeast vacuoles in vitro and in vivo $[75,80]$. Hence, the presence of these voluminous complexes can raise the interfacial free energy of the vertex by putting SNAREs into a 'higher gear', increasing the contact angle between the vacuoles and imposing a direct steric effect on the membranes.

\section{Conclusions and perspectives}

These considerations provide examples supporting the notion that the steric effects of fusion proteins and the geometric properties of the docking and fusion zone have an important impact on the energetics of the fusion process, which must be taken into account. Turning our attention to this aspect of SNARE-driven fusion reactions will allow us to uncover novel properties of the fusion machinery. It will require to take a more holistic view of this machinery, which does not only consist of SNARE complexes but involves several other highly conserved protein families, which associate with them. In physiological fusion reactions, these conserved SNARE-associated proteins are just as essential for fusion as SNAREs are, underlining that they have not only accessory functions in tethering or facilitation of SNARE complex formation, but that they also play major roles in the fusion reaction itself.

\section{Abbreviations}

MTCs, multi-subunit tether complexes; TMD, transmembrane domains.

\section{Acknowledgements}

A.M. was supported by the Swiss National Science Foundation (31003A_179306). H.J.R is grateful for support from the NWO (NWO Vidi scheme, The Netherlands) and the life@nano excellence initiative of the State of Lower Saxony, Germany. 


\section{Competing Interests}

The authors declare that there are no competing interests associated with the manuscript.

\section{References}

1 Cohen, F.S. and Melikyan, G.B. (2004) The energetics of membrane fusion from binding, through hemifusion, pore formation, and pore enlargement. J. Membr. Biol 199, 1-14 https://doi.org/10.1007/s00232-004-0669-8

2 Chernomordik, L.V. and Kozlov, M.M. (2008) Mechanics of membrane fusion. Nat. Struct. Mol. Biol. 15, 675-683 https://doi.org/10.1038/nsmb.1455

3 Chernomordik, L.V., Zimmerberg, J. and Kozlov, M.M. (2006) Membranes of the world unite!. J. Cell Biol. 175, 201-207 https://doi.org/10.1083/jcb. 200607083

4 Efrat, A., Chernomordik, L.V. and Kozlov, M.M. (2007) Point-like protrusion as a prestalk intermediate in membrane fusion pathway. Biophys. J. 92, L61-L63 https://doi.org/10.1529/biophysj.106.103341

5 Chang, C.-W., Chiang, C.-W. and Jackson, M.B. (2017) Fusion pores and their control of neurotransmitter and hormone release. J. Gen. Physiol. 149, 301-322 https://doi.org/10.1085/jgp.201611724

6 Xu, Y., Zhang, F., Su, Z., McNew, J.A. and Shin, Y.-K. (2005) Hemifusion in SNARE-mediated membrane fusion. Nat. Struct. Mol. Biol. 12, 417-422 https://doi.org/10.1038/nsmb921

7 Giraudo, C.G., Hu, C., You, D., Slovic, A.M., Mosharov, E.V., Sulzer, D. et al. (2005) SNAREs can promote complete fusion and hemifusion as alternative outcomes. J. Cell Biol. 170, 249-260 https://doi.org/10.1083/jcb.200501093

8 Lu, X., Zhang, F., McNew, J.A. and Shin, Y.-K. (2005) Membrane fusion induced by neuronal SNAREs transits through hemifusion. J. Biol. Chem. 280 30538-30541 https://doi.org/10.1074/jbc.M506862200

9 Wong, J.L., Koppel, D.E., Cowan, A.E. and Wessel, G.M. (2007) Membrane hemifusion is a stable intermediate of exocytosis. Dev. Cell 12, 653-659 https://doi.org/10.1016/j.devcel.2007.02.007

10 Reese, C., Heise, F. and Mayer, A. (2005) Trans-SNARE pairing can precede a hemifusion intermediate in intracellular membrane fusion. Nature 436, 410-414 https://doi.org/10.1038/nature03722

11 Lindau, M. and Almers, W. (1995) Structure and function of fusion pores in exocytosis and ectoplasmic membrane fusion. Curr. Opin. Cell Biol. 7, 509-517 https://doi.org/10.1016/0955-0674(95)80007-7

12 Han, X. and Jackson, M.B. (2005) Electrostatic interactions between the syntaxin membrane anchor and neurotransmitter passing through the fusion pore. Biophys. J. 88, L20-L22 https://doi.org/10.1529/biophysj.104.056739

13 Chang, C.-W., Hui, E., Bai, J., Bruns, D., Chapman, E.R. and Jackson, M.B. (2015) A structural role for the synaptobrevin 2 transmembrane domain in dense-core vesicle fusion pores. J. Neurosci. 35, 5772-5780 https://doi.org/10.1523/JNEUROSCl.3983-14.2015

14 Peters, C., Bayer, M.J., Bühler, S., Andersen, J.S., Mann, M. and Mayer, A. (2001) Trans-complex formation by proteolipid channels in the terminal phase of membrane fusion. Nature $\mathbf{4 0 9}, 581-588 \mathrm{https}: / /$ doi.org/10.1038/35054500

15 Strasser, B., Iwaszkiewicz, J., Michielin, 0. and Mayer, A. (2011) The V-ATPase proteolipid cylinder promotes the lipid-mixing stage of SNARE-dependent fusion of yeast vacuoles. EMBO J. 30, 4126-4141 https://doi.org/10.1038/emboj.2011.335

16 Risselada, H.J., Bubnis, G. and Grubmüller, H. (2014) Expansion of the fusion stalk and its implication for biological membrane fusion. Proc. Natl Acad. Sci. U.S.A. 111, 11043-11048 https://doi.org/10.1073/pnas.1323221111

17 Risselada, H.J. and Grubmüller, H. (2012) How SNARE molecules mediate membrane fusion: recent insights from molecular simulations. Curr. Opin. Struct. Biol. 22, 187-196 https://doi.org/10.1016/j.sbi.2012.01.007

18 Ryham, R.J., Klotz, T.S., Yao, L. and Cohen, F.S. (2016) Calculating transition energy barriers and characterizing activation states for steps of fusion. Biophys. J. 110, 1110-1124 https://doi.org/10.1016/j.bpj.2016.01.013

19 Gillingham, A.K. and Munro, S. (2019) Transport carrier tethering - how vesicles are captured by organelles. Curr. Opin. Cell Biol. 59, 140-146 https://doi.org/10.1016/j.ceb.2019.04.010

20 Wong, M. and Munro, S. (2014) Membrane trafficking. The specificity of vesicle traffic to the Golgi is encoded in the golgin coiled-coil proteins. Science 346, $1256898 \mathrm{https}: / /$ doi.org/10.1126/science. 1256898

21 Ungermann, C. and Kümmel, D. (2019) Structure of membrane tethers and their role in fusion. Traffic 20, 479-490 https://doi.org/10.1111/tra.12655

22 Baker, R.W. and Hughson, F.M. (2016) Chaperoning SNARE assembly and disassembly. Nat. Rev. Mol. Cell Biol. 17, 465-479 https://doi.org/10.1038/ nrm.2016.65

23 McBride, H.M., Rybin, V., Murphy, C., Giner, A., Teasdale, R. and Zerial, M. (1999) Oligomeric complexes link Rab5 effectors with NSF and drive membrane fusion via interactions between EEA1 and syntaxin 13. Cel/ 98, 377-386 https://doi.org/10.1016/S0092-8674(00)81966-2

24 Ren, Y., Yip, C.K., Tripathi, A., Huie, D., Jeffrey, P.D., Walz, T. et al. (2009) A structure-based mechanism for vesicle capture by the multisubunit tethering complex Dsl1. Cell 139, 1119-1129 https://doi.org/10.1016/j.cell.2009.11.002

25 Lobingier, B.T. and Merz, A.J. (2012) Sec1/Munc18 protein Vps33 binds to SNARE domains and the quaternary SNARE complex. Mol. Biol. Cell 23, 4611-4622 https://doi.org/10.1091/mbc.e12-05-0343

26 Peng, R. and Gallwitz, D. (2002) Sly1 protein bound to Golgi syntaxin Sed5p allows assembly and contributes to specificity of SNARE fusion complexes. J. Cell Biol. 157, 645-655 https://doi.org/10.1083/jcb.200202006

27 Dulubova, I., Khvotchev, M., Liu, S., Huryeva, I., Südhof, T.C. and Rizo, J. (2007) Munc18-1 binds directly to the neuronal SNARE complex. Proc. Natl Acad. Sci. U.S.A. 104, 2697-2702 https://doi.org/10.1073/pnas.0611318104

28 Orr, A., Song, H., Rusin, S.F., Kettenbach, A.N. and Wickner, W. (2017) HOPS catalyzes the interdependent assembly of each vacuolar SNARE into a SNARE complex. Mol. Biol. Cell 28, 975-983 https://doi.org/10.1091/mbc.e16-10-0743

29 Jiao, J., He, M., Port, S.A., Baker, R.W., Xu, Y., Qu, H., et al. (2018) Munc18-1 catalyzes neuronal SNARE assembly by templating SNARE association. eLife 7, e41771 https://doi.org/10.7554/eLife.41771

30 Baker, R.W., Jeffrey, P.D., Zick, M., Phillips, B.P., Wickner, W.T. and Hughson, F.M. (2015) A direct role for the Sec1/Munc18-family protein Vps33 as a template for SNARE assembly. Science $\mathbf{3 4 9}, 1111-1114 \mathrm{https}: / /$ doi.org/10.1126/science.aac7906

$31 \mathrm{Ma}, \mathrm{C} ., \mathrm{Su}, \mathrm{L} .$, Seven, A.B., Xu, Y. and Rizo, J. (2013) Reconstitution of the vital functions of Munc18 and Munc13 in neurotransmitter release. Science 339, 421-425 https://doi.org/10.1126/science.1230473 
32 Yu, H., Shen, C., Liu, Y., Menasche, B.L., Ouyang, Y., Stowell, M.H.B. et al. (2018) SNARE zippering requires activation by SNARE-like peptides in Sec1/ Munc18 proteins. Proc. Natl Acad. Sci. U.S.A. 115, E8421-E8429 https://doi.org/10.1073/pnas.1802645115

33 Collins, K.M., Thorngren, N.L., Fratti, R.A. and Wickner, W.T. (2005) Sec17p and HOPS, in distinct SNARE complexes, mediate SNARE complex disruption or assembly for fusion. EMBO J. 24, 1775-1786 https://doi.org/10.1038/sj.emboj.7600658

34 Lobingier, B.T., Nickerson, D.P., Lo, S.-Y. and Merz, A.J. (2014) SM proteins Sly1 and Vps33 co-assemble with Sec17 and SNARE complexes to oppose SNARE disassembly by Sec18. eLife $\mathbf{3}$, e02272 https://doi.org/10.7554/eLife.02272

35 Starai, V.J., Hickey, C.M. and Wickner, W. (2008) HOPS proofreads the trans-SNARE complex for yeast vacuole fusion. Mol. Biol. Cell 19, 2500-2508 https://doi.org/10.1091/mbc.e08-01-0077

36 Min, D., Kim, K., Hyeon, C., Hoon Cho, Y., Shin, Y.-K. and Yoon, T.-Y. (2013) Mechanical unzipping and rezipping of a single SNARE complex reveals hysteresis as a force-generating mechanism. Nat. Commun. 4, 1705 https://doi.org/10.1038/ncomms2692

37 Gao, Y., Zorman, S., Gundersen, G., Xi, Z., Ma, L., Sirinakis, G. et al. (2012) Single reconstituted neuronal SNARE complexes zipper in three distinct stages. Science 337, 1340-1343 https://doi.org/10.1126/science.1224492

38 Abdulreda, M.H., Bhalla, A., Rico, F., Berggren, P.-O., Chapman, E.R. and Moy, V.T. (2009) Pulling force generated by interacting SNAREs facilitates membrane hemifusion. Integr. Biol. (Camb) 1, 301-310 https://doi.org/10.1039/b900685k

39 Li, F., Pincet, F., Perez, E., Eng, W.S., Melia, T.J., Rothman, J.E. et al. (2007) Energetics and dynamics of SNAREpin folding across lipid bilayers. Nat. Struct. Mol. Biol. 14, 890-896 https://doi.org/10.1038/nsmb1310

40 Grote, E., Baba, M., Ohsumi, Y. and Novick, P.J. (2000) Geranylgeranylated SNAREs are dominant inhibitors of membrane fusion. J. Cell Biol. 151, 453-466 https://doi.org/10.1083/jcb.151.2.453

41 McNew, J.A., Weber, T., Parlati, F., Johnston, R.J., Melia, T.J., Söllner, T.H. et al. (2000) Close is not enough: SNARE-dependent membrane fusion requires an active mechanism that transduces force to membrane anchors. J. Cell Biol. 150, 105-117 https://doi.org/10.1083/jcb.150.1.105

42 Rohde, J., Dietrich, L., Langosch, D. and Ungermann, C. (2003) The transmembrane domain of Vam3 affects the composition of cis- and trans-SNARE complexes to promote homotypic vacuole fusion. J. Biol. Chem. 278, 1656-1662 https://doi.org/10.1074/jbc.M209522200

43 Pieren, M., Desfougères, Y., Michaillat, L., Schmidt, A. and Mayer, A. (2015) Vacuolar SNARE protein transmembrane domains serve as nonspecific membrane anchors with unequal roles in lipid mixing. J. Biol. Chem. 290, 12821-12832 https://doi.org/10.1074/jbc.M115.647776

44 Chang, C.-W., Chiang, C.-W., Gaffaney, J.D., Chapman, E.R. and Jackson, M.B. (2015) Lipid-anchored synaptobrevin provides little or no support for exocytosis or liposome fusion. J. Biol. Chem. 291, 2848-2857 https://doi.org/10.1074/jbc.M115.701169

45 Xu, H., Zick, M., Wickner, W.T. and Jun, Y. (2011) A lipid-anchored SNARE supports membrane fusion. Proc. Natl Acad. Sci. U.S.A. 108, 17325-17330 https://doi.org/10.1073/pnas.1113888108

46 D'Agostino, M., Risselada, H.J. and Mayer, A. (2016) Steric hindrance of SNARE transmembrane domain organization impairs the hemifusion-to-fusion transition. EMBO Rep. 17, 1590-1608 https://doi.org/10.15252/embr.201642209

47 Lakomek, N.-A., Yavuz, H., Jahn, R. and Pérez-Lara, A. (2019) Structural dynamics and transient lipid binding of synaptobrevin-2 tune SNARE assembly and membrane fusion. Proc. Natl Acad. Sci. U.S.A. 116, 8699-8708 https://doi.org/10.1073/pnas.1813194116

48 Lou, X. and Shin, Y.-K. (2016) SNARE zippering. Biosci. Rep. 36, e00327 https://doi.org/10.1042/BSR20160004

49 Borisovska, M., Schwarz, Y.N., Dhara, M., Yarzagaray, A., Hugo, S., Narzi, D., et al. (2012) Membrane-proximal tryptophans of synaptobrevin II stabilize priming of secretory vesicles. J. Neurosci. 32, 15983-15997 https://doi.org/10.1523/JNEUROSCl.6282-11.2012

50 Han, J., Pluhackova, K., Bruns, D. and Böckmann, R.A. (2016) Synaptobrevin transmembrane domain determines the structure and dynamics of the SNARE motif and the linker region. Biochim. Biophys. Acta 1858, 855-865 https://doi.org/10.1016/j.bbamem.2016.01.030

51 Lai, Y., Choi, U.B., Leitz, J., Rhee, H.J., Lee, C., Altas, B. et al. (2017) Molecular mechanisms of synaptic vesicle priming by Munc13 and Munc18. Neuron 95, 591-607.e10 https://doi.org/10.1016/j.neuron.2017.07.004

52 Shen, C., Rathore, S.S., Yu, H., Gulbranson, D.R., Hua, R., Zhang, C. et al. (2015) The trans-SNARE-regulating function of Munc18-1 is essential to synaptic exocytosis. Nat. Commun. 6, 8852 https://doi.org/10.1038/ncomms9852

53 Parisotto, D., Pfau, M., Scheutzow, A., Wild, K., Mayer, M.P., Malsam, J. et al. (2014) An extended helical conformation in domain 3a of Munc18-1 provides a template for SNARE (soluble N-ethylmaleimide-sensitive factor attachment protein receptor) complex assembly. J. Biol. Chem. 289 9639-9650 https://doi.org/10.1074/jbc.M113.514273

54 Lou, X., Shin, J., Yang, Y., Kim, J. and Shin, Y.-K. (2015) Synaptotagmin-1 is an antagonist for Munc18-1 in SNARE zippering. J. Biol. Chem. 290, 10535-10543 https://doi.org/10.1074/jbc.M114.631341

55 Stein, A., Weber, G., Wahl, M.C. and Jahn, R. (2009) Helical extension of the neuronal SNARE complex into the membrane. Nature 460, 525-528 https://doi.org/10.1038/nature08156

56 Hastoy, B., Scotti, P.A., Milochau, A., Fezoua-Boubegtiten, Z., Rodas, J., Megret, R., et al. (2017) A central small amino acid in the VAMP2 transmembrane domain regulates the fusion pore in exocytosis. Sci. Rep. 7, $2835 \mathrm{https}: / / \mathrm{doi} .0 \mathrm{rg} / 10.1038 / \mathrm{s} 41598-017-03013-3$

57 Han, X., Wang, C.-T., Bai, J., Chapman, E.R. and Jackson, M.B. (2004) Transmembrane segments of syntaxin line the fusion pore of $\mathrm{Ca}^{2+}$-triggered exocytosis. Science 304, 289-292 https://doi.org/10.1126/science.1095801

58 Chang, C.-W. and Jackson, M.B. (2015) Synaptobrevin transmembrane domain influences exocytosis by perturbing vesicle membrane curvature. Biophys. J. 109, 76-84 https://doi.org/10.1016/j.bpj.2015.05.021

59 Shi, L., Shen, Q.-T., Kiel, A., Wang, J., Wang, H.-W., Melia, T.J. et al. (2012) SNARE proteins: one to fuse and three to keep the nascent fusion pore open. Science 335, 1355-1359 https://doi.org/10.1126/science.1214984

60 Smirnova, Y.G., Risselada, H.J. and Müller, M. (2019) Thermodynamically reversible paths of the first fusion intermediate reveal an important role for membrane anchors of fusion proteins. Proc. Natl Acad. Sci. U.S.A. 116, 2571-2576 https://doi.org/10.1073/pnas.1818200116

61 Ngatchou, A.N., Kisler, K., Fang, Q., Walter, A.M., Zhao, Y., Bruns, D. et al. (2010) Role of the synaptobrevin C terminus in fusion pore formation. Proc. Natl Acad. Sci. U.S.A. 107, 18463-18468 https://doi.org/10.1073/pnas.1006727107

62 Wehland, J.-D., Lygina, A.S., Kumar, P., Guha, S., Hubrich, B.E., Jahn, R. et al. (2016) Role of the transmembrane domain in SNARE protein mediated membrane fusion: peptide nucleic acid/peptide model systems. Mol. Biosyst. 12, 2770-2776 https://doi.org/10.1039/C6MB00294C

63 Carr, C.M. and Rizo, J. (2010) At the junction of SNARE and SM protein function. Curr. Opin. Cell Biol. 22, 488-495 https://doi.org/10.1016/j.ceb. 2010.04.006 
64 Fisher, R.J., Pevsner, J. and Burgoyne, R.D. (2001) Control of fusion pore dynamics during exocytosis by Munc18. Science 291, 875-878 https://doi. org/10.1126/science.291.5505.875

65 Ciufo, L.F., Barclay, J.W., Burgoyne, R.D. and Morgan, A. (2005) Munc18-1 regulates early and late stages of exocytosis via syntaxin-independent protein interactions. Mol. Biol. Cell 16, 470-482 https://doi.org/10.1091/mbc.E04-08-0685

66 Boyd, A., Ciufo, L.F., Barclay, J.W., Graham, M.E., Haynes, L.P., Doherty, M.K. et al. (2008) A random mutagenesis approach to isolate dominant-negative yeast sec1 mutants reveals a functional role for domain 3a in yeast and mammalian Sec1/Munc18 proteins. Genetics 180, 165-178 https://doi.org/10.1534/genetics.108.090423

67 Zanetti, M.N., Bello, O.D., Wang, J., Coleman, J., Cai, Y., Sindelar, C.V. et al. (2016) Ring-like oligomers of Synaptotagmins and related C2 domain proteins. eLife 5, 947 https://doi.org/10.7554/eLife.17262

68 Rizo, J., Chen, X. and Araç, D. (2006) Unraveling the mechanisms of synaptotagmin and SNARE function in neurotransmitter release. Trends. Cell Biol. 16. 339-350 https://doi.org/10.1016/.t.tcb.2006.04.006

69 Li, X., Radhakrishnan, A., Grushin, K., Kasula, R., Chaudhuri, A., Gomathinayagam, S. et al. (2019) Symmetrical organization of proteins under docked synaptic-vesicles. FEBS Lett. 593, 144-153 https://doi.org/10.1002/1873-3468.13316

70 Bello, O.D., Jouannot, O., Chaudhuri, A., Stroeva, E., Coleman, J., Volynski, K.E. et al. (2018) Synaptotagmin oligomerization is essential for calcium control of regulated exocytosis. Proc. Natl Acad. Sci. U.S.A. 115, E7624-E7631 https://doi.org/10.1073/pnas.1808792115

71 Hickey, C.M. and Wickner, W. (2010) HOPS initiates vacuole docking by tethering membranes before trans-SNARE complex assembly. Mol. Biol. Cell 21, 2297-2305 https://doi.org/10.1091/mbc.e10-01-0044

72 Orr, A., Wickner, W., Rusin, S.F., Kettenbach, A.N. and Zick, M. (2015) Yeast vacuolar HOPS, regulated by its kinase, exploits affinities for acidic lipids and Rab:GTP for membrane binding and to catalyze tethering and fusion. Mol. Biol. Cell 26, 305-315 https://doi.org/10.1091/mbc. E14-08-1298

73 Cabrera, M., Ostrowicz, C.W., Mari, M., LaGrassa, T.J., Reggiori, F. and Ungermann, C. (2009) Vps41 phosphorylation and the Rab Ypt7 control the targeting of the HOPS complex to endosome-vacuole fusion sites. Mol. Biol. Cell 20, 1937-1948 https://doi.org/10.1091/mbc.e08-09-0943

74 Bröcker, C., Kuhlee, A., Gatsogiannis, C., Balderhaar, H.J.K., Hönscher, C., Engelbrecht-Vandré, S. et al. (2012) Molecular architecture of the multisubunit homotypic fusion and vacuole protein sorting (HOPS) tethering complex. Proc. Natl Acad. Sci. U.S.A. 109, 1991-1996 https://doi.org/10. 1073/pnas.1117797109

75 D'Agostino, M., Risselada, H.J., Lürick, A., Ungermann, C. and Mayer, A. (2017) A tethering complex drives the terminal stage of SNARE-dependent membrane fusion. Nature $\mathbf{5 5 1}, 634-638$ https://doi.org/10.1038/nature24469

76 Wang, L., Seeley, E.S., Wickner, W. and Merz, A.J. (2002) Vacuole fusion at a ring of vertex docking sites leaves membrane fragments within the organelle. Cell 108, 357-369 https://doi.org/10.1016/S0092-8674(02)00632-3

77 Fratti, R.A., Jun, Y., Merz, A.J., Margolis, N. and Wickner, W. (2004) Interdependent assembly of specific regulatory lipids and membrane fusion proteins into the vertex ring domain of docked vacuoles. J. Cell Biol. 167, 1087-1098 https://doi.org/10.1083/jcb.200409068

78 Karunakaran, S., Sasser, T., Rajalekshmi, S. and Fratti, R.A. (2012) SNAREs, HOPS, and regulatory lipids control the dynamics of vacuolar actin during homotypic fusion. J. Cell Sci. 125, 1683-1692 https://doi.org/10.1242/jcs.091900

79 Brandt, T., Cavellini, L., Kühlbrandt, W. and Cohen, M.M. (2016) A mitofusin-dependent docking ring complex triggers mitochondrial fusion in vitro. eLife 5, e14618 https://doi.org/10.7554/eLife.14618

80 D'Agostino, M., Risselada, H.J., Endter, L.J., Comte-Miserez, V. and Mayer, A. (2018) SNARE-mediated membrane fusion arrests at pore expansion to regulate the volume of an organelle. EMBO J. 37, e99193 https://doi.org/10.15252/embj.201899193

81 Mostafavi, H., Thiyagarajan, S., Stratton, B.S., Karatekin, E., Warner, J.M., Rothman, J.E. et al. (2017) Entropic forces drive self-organization and membrane fusion by SNARE proteins. Proc. Natl Acad. Sci. U.S.A. 114, 5455-5460 https://doi.org/10.1073/pnas.1611506114

82 Lindén, M., Sens, P. and Phillips, R. (2012) Entropic tension in crowded membranes. PLOS Comput. Biol. 8, e1002431 https://doi.org/10.1371/journal. pcbi.1002431

83 Ryham, R.J., Ward, M.A. and Cohen, F.S. (2013) Teardrop shapes minimize bending energy of fusion pores connecting planar bilayers. Phys. Rev. E 88 693 https://doi.org/10.1103/PhysRevE.88.062701

84 Pieren, M., Schmidt, A. and Mayer, A. (2010) The SM protein Vps33 and the t-SNARE H(abc) domain promote fusion pore opening. Nat. Struct. Mol. Biol. 17, 710-717 https://doi.org/10.1038/nsmb.1809

85 Karatekin, E. (2018) Toward a unified picture of the exocytotic fusion pore. FEBS Lett. 592, 3563-3585 https://doi.org/10.1002/1873-3468.13270

86 Bao, H., Das, D., Courtney, N.A., Jiang, Y., Briguglio, J.S., Lou, X. et al. (2018) Dynamics and number of trans-SNARE complexes determine nascent fusion pore properties. Nature 554, 260-263 https://doi.org/10.1038/nature25481

87 Zhao, W.-D., Hamid, E., Shin, W., Wen, P.J., Krystofiak, E.S., Villarreal, S.A. et al. (2016) Hemi-fused structure mediates and controls fusion and fission in live cells. Nature 534, 548-552 https://doi.org/10.1038/nature18598

88 Kweon, D.-H., Kong, B. and Shin, Y.-K. (2017) Hemifusion in synaptic vesicle cycle. Front. Mol. Neurosci. 10, 65 https://doi.org/10.3389/fnmol.2017. 00065

89 Chizmadzhev, Y.A., Kumenko, D.A., Kuzmin, P.I., Chernomordik, L.V., Zimmerberg, J. and Cohen, F.S. (1999) Lipid flow through fusion pores connecting membranes of different tensions. Biophys. J. 76, 2951-2965 https://doi.org/10.1016/S0006-3495(99)77450-3

90 Chizmadzhev, Y.A., Kuzmin, P.I., Kumenko, D.A., Zimmerberg, J. and Cohen, F.S. (2000) Dynamics of fusion pores connecting membranes of different tensions. Biophys. J. 78, 2241-2256 https://doi.org/10.1016/S0006-3495(00)76771-3

91 Bretou, M., Jouannot, O., Fanget, I., Pierobon, P., Larochette, N., Gestraud, P., et al. (2014) Cdc42 controls the dilation of the exocytotic fusion pore by regulating membrane tension. Mol. Biol. Cell 25, 3195-3209 https://doi.org/10.1091/mbc.e14-07-1229

92 Cohen, F.S., Zimmerberg, J. and Finkelstein, A. (1980) Fusion of phospholipid vesicles with planar phospholipid bilayer membranes. II. Incorporation of a vesicular membrane marker into the planar membrane. J. Gen. Physiol. 75, 251-270 https://doi.org/10.1085/jgp.75.3.251

93 Tran, D.T., Masedunskas, A., Weigert, R., Hagen, T. and G, K. (2015) Arp2/3-mediated F-actin formation controls regulated exocytosis in vivo. Nat. Commun. 6, 10098 https://doi.org/10.1038/ncomms 10098

94 Rousso, T., Schejter, E.D. and Shilo, B.-Z. (2016) Orchestrated content release from Drosophila glue-protein vesicles by a contractile actomyosin network. Nat. Cell Biol. 18, 181-190 https://doi.org/10.1038/ncb3288 
95 Wu, Z., Bello, O.D., Thiyagarajan, S., Auclair, S.M., Vennekate, W., Krishnakumar, S.S. et al. (2017) Dilation of fusion pores by crowding of SNARE proteins. elife 6, e22964 https://doi.org/10.7554/eLife.22964

96 Laage, D., Elsaesser, T. and Hynes, J.T. (2017) Water dynamics in the hydration shells of biomolecules. Chem. Rev. 117, 10694-10725 https://doi. org/10.1021/acs.chemrev.6b00765

97 Smirnova, Y.G., Aeffner, S., Risselada, H.J., Salditt, T., Marrink, S.J., Muller, M. et al. (2013) Interbilayer repulsion forces between tension-free lipid bilayers from simulation. Soft Matter $\mathbf{9}, 10705-10718 \mathrm{https}: / /$ doi.org/10.1039/c3sm51771c

98 Pannuzzo, M., McDargh, Z.A. and Deserno, M. (2018) The role of scaffold reshaping and disassembly in dynamin driven membrane fission. elife 7 , $2270 \mathrm{https}: / /$ doi.org/10.7554/eLife.39441

99 Sharma, S. and Lindau, M. (2018) Molecular mechanism of fusion pore formation driven by the neuronal SNARE complex. Proc. Natl Acad. Sci. U.S.A. 115, 12751-12756 https://doi.org/10.1073/pnas.1816495115

100 Fernández-Busnadiego, R., Zuber, B., Maurer, U.E., Cyrklaff, M., Baumeister, W. and Lucic, V. (2010) Quantitative analysis of the native presynaptic cytomatrix by cryoelectron tomography. J. Cell Biol. 188, 145-156 https://doi.org/10.1083/jcb.200908082

101 Imig, C., Min, S.-W., Krinner, S., Arancillo, M., Rosenmund, C., Südhof, T.C. et al. (2014) The morphological and molecular nature of synaptic vesicle priming at presynaptic active zones. Neuron 84, 416-431 https://doi.org/10.1016/j.neuron.2014.10.009

102 Risselada, H.J., Smirnova, Y. and Grubmüller, H. (2014) Free energy landscape of rim-pore expansion in membrane fusion. Biophys. J. 107, 2287-2295 https://doi.org/10.1016/j.bpj.2014.08.022

103 Mayer, A. and Wickner, W. (1997) Docking of yeast vacuoles is catalyzed by the Ras-like GTPase Ypt7p after symmetric priming by Sec18p (NSF). J. Cell Biol. 136, 307-317 https://doi.org/10.1083/jcb.136.2.307

104 Ungermann, C., Sato, K. and Wickner, W. (1998) Defining the functions of trans-SNARE pairs. Nature 396, 543-548 https://doi.org/10.1038/25069

105 Eitzen, G., Wang, L., Thorngren, N. and Wickner, W. (2002) Remodeling of organelle-bound actin is required for yeast vacuole fusion. J. Cell Biol. 158, 669-679 https://doi.org/10.1083/jcb.200204089

106 Eitzen, G., Thorngren, N. and Wickner, W. (2001) Rho1p and Cdc42p act after Ypt7p to regulate vacuole docking. EMBO J. 20, 5650-5656 https://doi. org/10.1093/emboj/20.20.5650

107 Isgandarova, S., Jones, L., Forsberg, D., Loncar, A., Dawson, J., Tedrick, K. et al. (2007) Stimulation of actin polymerization by vacuoles via Cdc42p-dependent signaling. J. Biol. Chem. 282, 30466-30475 https://doi.org/10.1074/jbc.M704117200

108 Chou, H.-T., Dukovski, D., Chambers, M.G., Reinisch, K.M. and Walz, T. (2016) CATCHR, HOPS and CORVET tethering complexes share a similar architecture. Nat. Struct. Mol. Biol. 23, 761-763 https://doi.org/10.1038/nsmb.3264

109 Baker, R.W., Jeffrey, P.D. and Hughson, F.M. (2013) Crystal structures of the Sec1/Munc18 (SM) protein Vps33, alone and bound to the homotypic fusion and vacuolar protein sorting (HOPS) subunit Vps16*. PLoS One 8, e67409 https://doi.org/10.1371/journal.pone.0067409 